\title{
Synthesis, characterization and magnetic properties of $\mathrm{Ni}, \mathrm{Co}$ and $\mathrm{FeCo}$ nanoparticles on reduced graphene oxide for removal of $\mathrm{Cr}(\mathrm{VI})$
}

\author{
Nduduzo N. Malinga ${ }^{1}$ (D) A. L. Leigh Jarvis ${ }^{1}$
}

Received: 6 September 2019 / Accepted: 26 December 2019 / Published online: 23 January 2020

(c) The Author(s) 2020

\begin{abstract}
To remove pollutants from industrial waste, magnetic separation by use of magnetic reduced graphene oxide (rGO) is a possible route, due to the high specific surface area of rGO. Reduced graphene oxide decorated with nickel, cobalt and cobalt ferrite nanoparticles was synthesized by means of modified coprecipitation methods. Nitrogen-doped reduced graphene was prepared by a thermal doping method. The resulting composites were characterized with scanning electron microscope, transmission electron microscope (TEM), powder X-ray diffraction (XRD), thermal analysis and Raman spectroscopy. Samples were magnetically characterized using vibrating sample magnetometer to determine the magnetic properties. All the prepared sampled were found to have weak ferromagnetic properties. The particle size distribution of the nanoparticles was determined using the TEM images and Image $J$ software. The average particle size for the Co-rGO was $1.89 \mathrm{~nm}, 35.12 \mathrm{~nm}$ for $\mathrm{Ni}-\mathrm{rGO}$ and $32.15 \mathrm{~nm}$ for $\mathrm{CoFe}-\mathrm{rGO}$. The $\mathrm{Co}-\mathrm{rGO}$ was used as proof of principle to remove $\mathrm{Cr}(\mathrm{VI})$ ions from solution. The Co-rGO was recycled five times before it was deemed unusable.
\end{abstract}

Nduduzo N. Malinga

205500488@stu.ukzn.ac.za

Discipline of Electrical, Electronic and Computer

Engineering, University of KwaZulu-Natal, Howard College

Campus, Durban, South Africa 


\section{Graphic abstract}

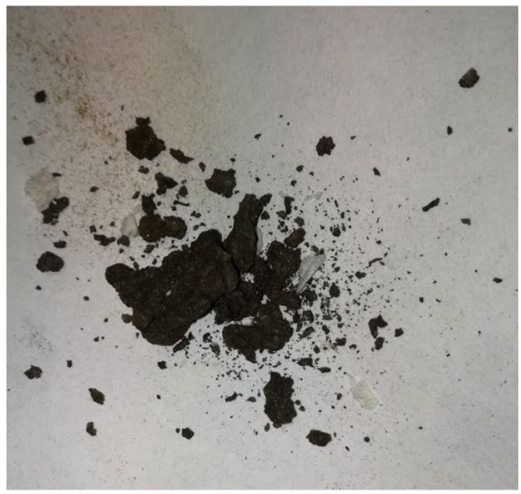

GO
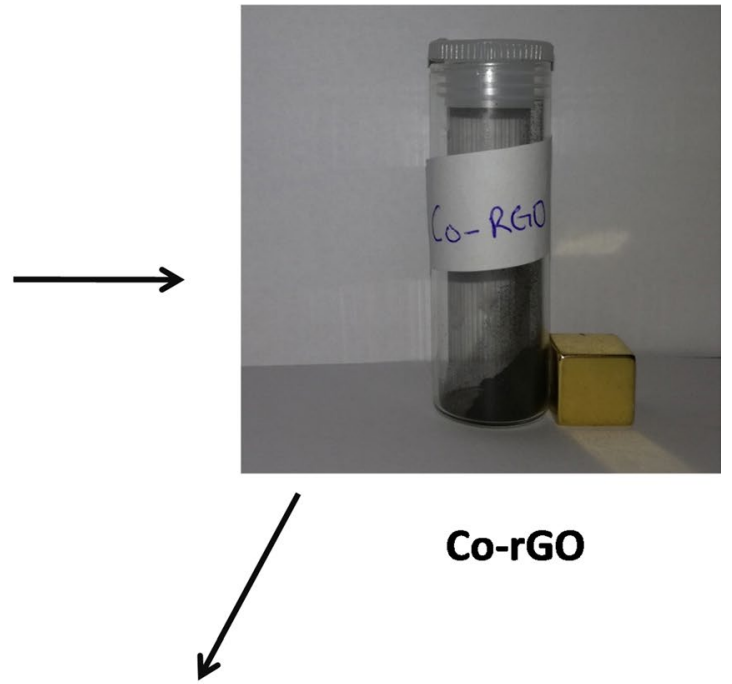

\section{Co-rGO}

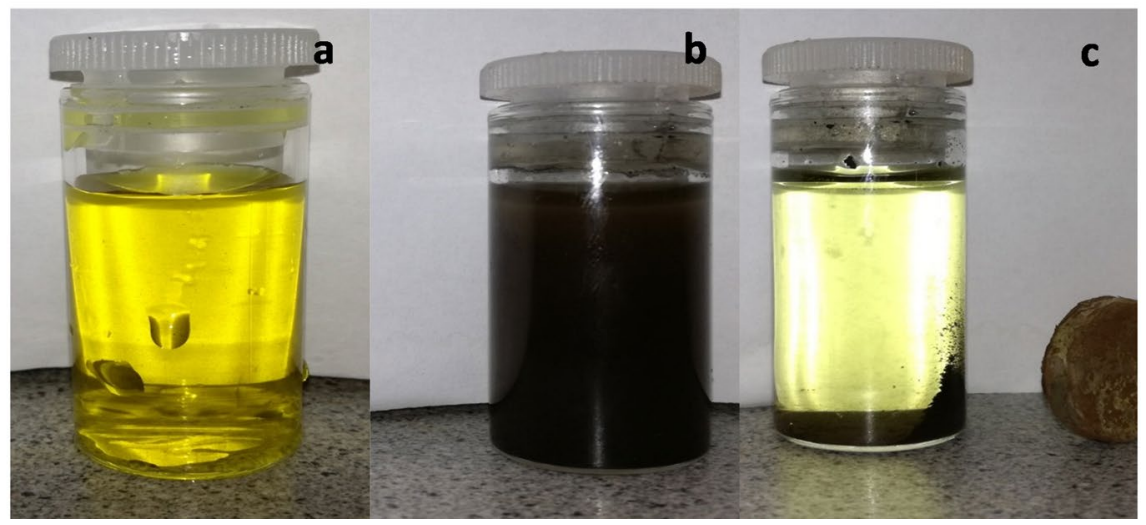

Keywords Magnetic properties $\cdot$ Magnetic nanoparticles $\cdot$ Graphene synthesis $\cdot$ Reduced graphene oxide

\section{Introduction}

Magnetic nanoparticles have received significant research attention since they were discovered two decades ago [1]. Interest was due to their magnetic properties, which depended on the size and shape of the particles [2]. The magnetic nanoparticles have been used in a variety of applications such as: wastewater treatment [3], medical imaging [4], and drug delivery [5]. Other nanoparticles exhibit antiferromagnetic behavior and have magnetization reversal using quantum tunneling [6]. The nanoparticles which are antiferromagnetic can exhibit superparamagnetic or ferromagnetic behavior as the particle size decreases [6].

Graphene has a high surface to volume ratio which allows it to be a suitable substrate to disperse nanoparticles. This leads to the formation of graphene nanoparticles which have been significantly studied in the past decade. The formation of graphene-nanoparticle composites has opened up new and different avenues of research and applications. The composites may have different magnetic properties as compared to the individual components which has been shown in graphene- $\mathrm{Co}_{3} \mathrm{O}_{4}$ composite, where the composite is superparamagnetic and the pure $\mathrm{Co}_{3} \mathrm{O}_{4}$ is antiferromagnetic [6]. Graphene cobalt-boron nanoparticles have shown to be promising candidates as the anode in lithium ion batteries. The electrode has been shown to have a high reversible capacity [7]. The other promising application of graphene-based compounds is graphene aerosol decorated with iron cobalt oxide nanoparticles in Li-ion batteries [8]. Composite graphene material has been shown to be useful as anode material for lithium ion batteries, where the nanoparticles prevent graphene sheet restacking. The composite has superior lithium ion storage, specific capacity and cycle stability [8]. Magnetic nanoparticles such as iron, cobalt and nickel have large saturation magnetization and high Snoek's limit and compatible dielectric loss [9]. The combination of 
the magnetic nanoparticle with graphene is beneficial. The large surface area is combined with dielectric loss, magnetic loss component, increased interface polarization and electromagnetic loss ability. This implies graphene-based compounds have microwave absorbance antenna performance [9]. The combination of graphene and nickel nanoparticles shows enhanced performance of microwave absorbance than graphene and nickel nanoparticles individually due to the improvement of electromagnetic loss [9]. Nitrogen-functionalized graphene nanosheet offers sites for loading of cobalt nanoparticles, which act as a catalyst for Fischer-Tropsch synthesis gas to liquid conversion [10]. Ruthenium can be added to nitrogen-doped reduced graphene cobalt nanoparticles composites, which promotes the activity of cobalt for better yields [10].

Titanium dioxide $\left(\mathrm{TiO}_{2}\right)$ nanoparticles are widely known to be semiconductors and photocatalysts $[11,12]$. The addition of graphene to $\mathrm{TiO}_{2}$ enhances the catalytic activity of $\mathrm{TiO}_{2}$, because graphene has high electron mobility and high surface area which is more stable to photobleaching than $\mathrm{TiO}_{2}$ on its own [11]. $\mathrm{TiO}_{2}-$ graphene has been useful in the degradation of volatile organic compounds. $\mathrm{The}^{\mathrm{TiO}_{2}}$-graphene oxide when dispersed in ethanol has been shown to reduce graphene oxide (GO) to reduced graphene oxide (rGO). The $\mathrm{TiO}_{2}-\mathrm{rGO}$ composite has a higher affinity to adsorb organic dyes, a higher charge separation and a larger charge transport potential $[11,12]$. The formation of graphene nanoparticle composite is very cost-effective because it requires one reaction vessel and produces a composite with superior conductivity over bare reduced graphene oxide [13]. The nanoparticles also prevent restacking of graphene sheets and reforming graphite. The method of in situ simultaneous reduction is very versatile in producing different shapes and sizes of the nanoparticles on graphene sheets which is the method of choice in this study [13]. Different shapes of nanoparticles are obtained using different stabilizing agents. The method also produces highly crystalline nanoparticles with a generally low particle size distribution.

Pristine graphene exhibits long-range ferromagnetic coupling $[14,15]$, and reduced graphene oxide (rGO) has weak ferromagnetic properties, which is caused by a zigzag pattern on the edge of the graphene. Doping of rGO with nitrogen has been shown to increase the magnetic properties [14].

In this study, we look at magnetic nanoparticles anchored on rGO, that is, nanocomposites. The magnetic composites could help in the retrieving and recycling of graphene after targeted adsorption of certain pollutants. Due to their magnetic uses in industry, the following nanoparticles were selected in this comparison study: cobalt, nickel and cobalt ferrite.

\section{Materials and methods}

\section{Material}

Phosphoric acid $85 \%\left(\mathrm{H}_{3} \mathrm{PO}_{4}\right)$, sulfuric acid $98 \%\left(\mathrm{H}_{2} \mathrm{SO}_{4}\right)$, potassium permanganate $\left(\mathrm{KMnO}_{4}\right) 99 \%$, ethanol $99.9 \%$ and hydrochloric acid $(\mathrm{HCl}) 35 \%$ were purchased from Associated Chemical Enterprise. Potassium platinate 98\% $\left(\mathrm{K}_{2} \mathrm{PtCl}_{4}\right)$, iron chloride $98 \%\left(\mathrm{FeCl}_{3}\right)$ and iron sulfate heptahydrate $\left(\mathrm{FeSO}_{4} \cdot 7 \mathrm{H}_{2} \mathrm{O}\right) 98 \%$ were purchased from SigmaAldrich. Cobalt acetate $98 \%$ (CoAc) was purchased from BDH Chemicals and nickel chloride $98 \%\left(\mathrm{NiCl}_{2}\right)$ was purchased from Saarchem. Expandable graphite (98\%) was donated by Richards Bay Minerals.

\section{Equipment}

The prepared samples were analyzed using the following techniques.

Infrared (IR) spectra were recorded on a Perkin Elmer Spectrum 100 ATR FT-IR spectrometer. X-ray powder diffraction (XRD) patterns were recorded on a PAN analytical, EMPYREAN, using a Co-K $\alpha$ monochromatic radiation with PIXcel detector. Data were collected in the range $2 \theta=5^{\circ}-90^{\circ}$, scanning at $2^{\circ} \mathrm{min}^{-1}$ with a filter time constant of $2.5 \mathrm{~s}$ per step and a slit width of $6.0 \mathrm{~mm}$. Transmission electron microscope (TEM) images were recorded on a JEOL JEM 1010 with the accelerating voltage of $18 \mathrm{kV}$. Scanning electron microscope (SEM) images were recorded using ZEISS Ultra Plus Field Emission Gun Scanning Electron Microscope. Thermogravimetric analysis (TGA) together with differential scanning calorimetry (DSC) was recorded on Q600 SDT TA Instruments. The samples were placed in a ceramic pan and measurements conducted under nitrogen flow $\left(20 \mathrm{~mL} \mathrm{~min}^{-1}\right)$ from room temperature to $1000{ }^{\circ} \mathrm{C}$ at a heating rate of $10{ }^{\circ} \mathrm{C} \mathrm{min}-1$ Magnetization experiments were recorded on the lakeshore magnetometer system $735 \mathrm{~S}$. The specimen was placed in a sample holder and stoppered with cotton wool to prevent the sample from having torque. The sample was then vibrated in the presence of a sweeping electromagnetic field from -15 to $15 \mathrm{kOe}$. The metal concentration of nanoparticle specimens was achieved by use of an inductively coupled plasma-optical emission spectroscopy (ICP-OES), Perkin Elmer Optima 5300 DV. WinLab 32 software was used to analyze the data. UV/Vis spectra were recorded using a Perkin Elmer Lambda $25 \mathrm{UV} / \mathrm{Vis}$ spectrophotometer. 


\section{Method}

\section{Synthesis of reduced graphene oxide $(\mathrm{rGO})$}

The graphene oxide was prepared according to the modified Tour's method [16]. Expandable graphite $(3.11 \mathrm{~g})$ was microwaved for $30 \mathrm{~s}$ yielding expanded graphite. We have observed that the microwave step helps in the exfoliation process and uses lesser amounts of oxidants. The graphite was then mixed with phosphoric acid $(30 \mathrm{~mL})$ in a $30 \mathrm{~mL}$ pill vial. The pill vial was sealed and placed in an ultrasonic bath and sonicated for $1 \mathrm{~h}$. The graphite was partially dispersed in the acid and transferred to a $500 \mathrm{~mL}$ roundbottom flask. The flask was cooled to $0{ }^{\circ} \mathrm{C}$ and sulfuric acid $(270 \mathrm{~mL})$ was added dropwise. The solution was stirred with the slow addition of potassium permanganate $(13.5 \mathrm{~g})$. The solution was stirred for $12 \mathrm{~h}$. The solution was then cooled to room temperature, followed by the addition of ice $(400 \mathrm{~mL})$, calcium carbonate, sodium bicarbonate and $\mathrm{H}_{2} \mathrm{O}_{2}(3 \mathrm{~mL})$. The mixture was sifted through a stainless steel sieve (Clear Edge testing sieve, $300 \mu \mathrm{m}$ ) and the filtrate was collected. The filtrate was then centrifuged to collect the solid product, which was washed twice with deionized water $(200 \mathrm{~mL})$, $\mathrm{HCl}(200 \mathrm{~mL})$ and ethanol $(200 \mathrm{~mL})$. A final third wash was carried out with water $(200 \mathrm{~mL})$, ethanol $(200 \mathrm{~mL})$ and diethyl ether $(200 \mathrm{~mL})$. Graphene oxide $(0.5 \mathrm{~g})$ was then mixed with $25 \mathrm{ml}$ deionized water and ultrasonicated until the graphene oxide was well dispersed and formed a dark brown solution. The solution was transferred into a $250 \mathrm{~mL}$ volumetric flask. To the solution, a solution of sodium borohydride $0.04 \mathrm{M}(10 \mathrm{~mL})$ was added dropwise and refluxed for $30 \mathrm{~min}$. The color changed from brown to black, indicating the reduction of graphene oxide.

\section{Synthesis of nitrogen-doped reduced graphene oxide (N-rGO)}

Nitrogen doped reduced graphene oxide was synthesized by the decomposition of graphene oxide solution with urea according to a modified literature method [15]. Graphene oxide $(0.5 \mathrm{~g})$ was mixed with ethanolamine $(2 \mathrm{ml})$, deionized water $(2 \mathrm{~mL})$ and urea $(0.2 \mathrm{~g})$ in a pill vial. The mixture was sonicated until homogenous and had no particulate. The thick mixture was then transferred to a tantalum boat and heated in a tubular furnace under an inert atmosphere. The solution mixture was heated to $700{ }^{\circ} \mathrm{C}$ at a heating rate of $5{ }^{\circ} \mathrm{C} \min ^{-1}$ and pyrolyzed for $1 \mathrm{~h}$. The furnace was then cooled to room temperature still under inert conditions. The product obtained was washed with near boiling water $(4 \times 100 \mathrm{~mL})$ to remove unwanted ions followed by ethanol.
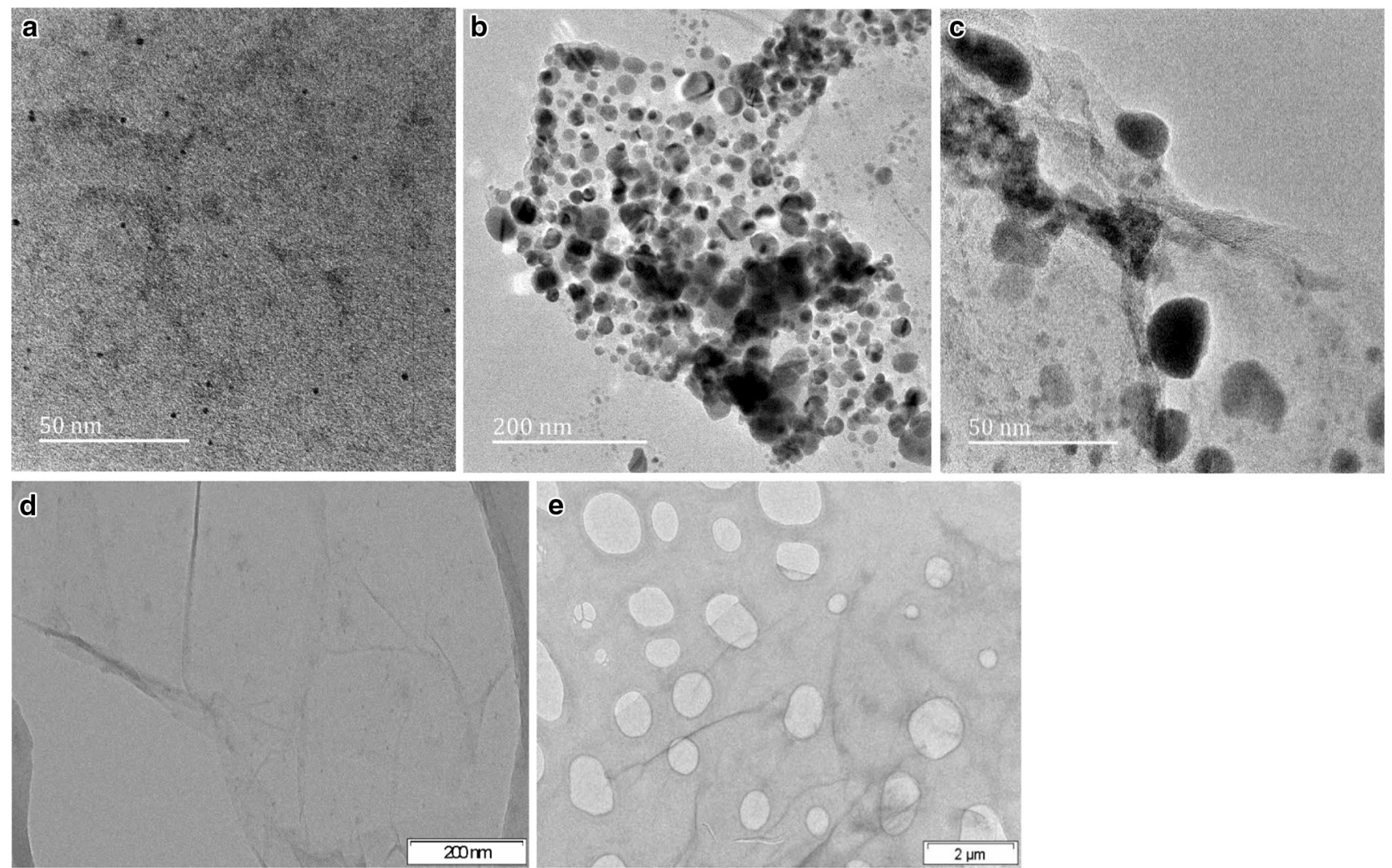

Fig. 1 TEM images of a Co-rGO, b Ni-rGO, $\mathbf{c}$ FeCo-rGO, $\mathbf{d}$ rGO, e N-rGO 

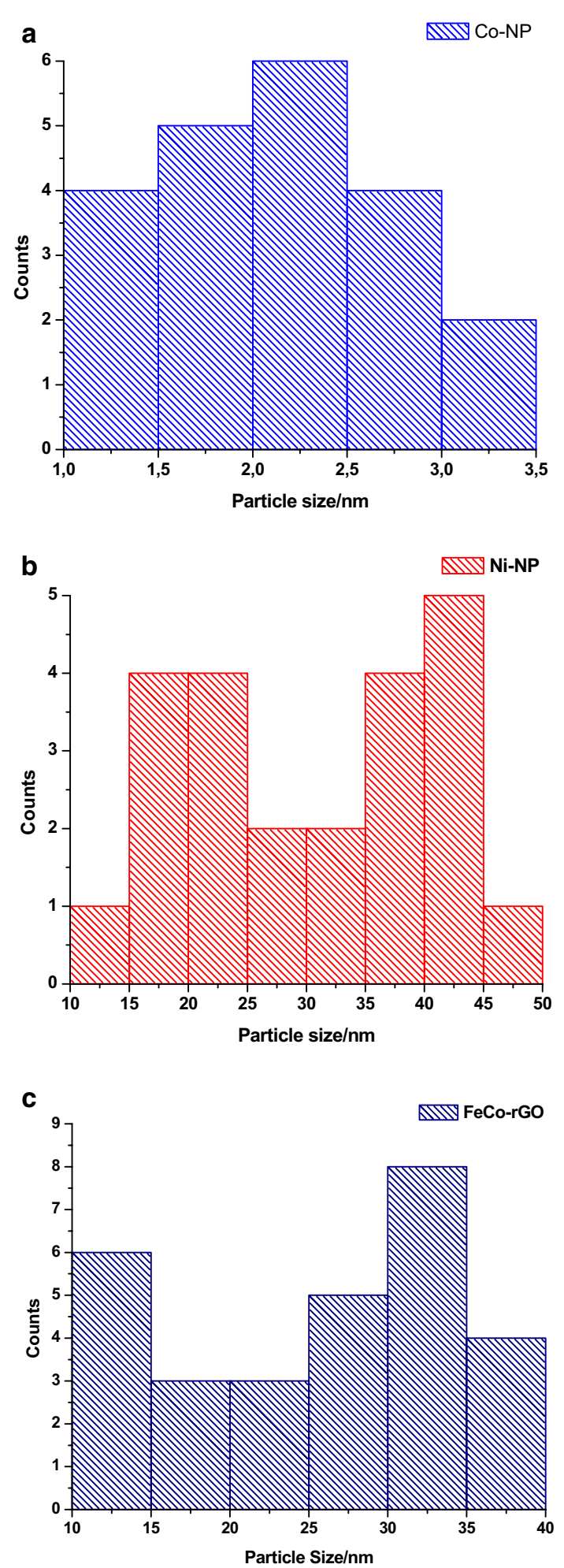

Fig. 2 Particle size distribution of a Co-Np, b Ni-NP and $\mathbf{c}$ FeCoONP on rGO

The colloidal powder was dried in a vacuum oven at $60^{\circ} \mathrm{C}$. The reduced graphene oxide was synthesized in a similar manner without the addition of urea.
Synthesis of graphene decorated with cobalt nanoparticles (Co-rGO)

The reduced graphene oxide-cobalt nanoparticle composite was synthesized according to the modified literature method $[17,18]$. Graphene oxide $(1.01 \mathrm{~g})$ was mixed with deionized water $(25 \mathrm{~mL})$ and ultrasonicated until the graphene oxide was well dispersed and formed a dark brown solution. The solution was transferred into a $250 \mathrm{~mL}$ three-neck volumetric flask. Cobalt acetate $(2 \mathrm{~g})$ and potassium hydroxide $(0.05 \mathrm{~g})$ were weighed into the solution and refluxed at $90{ }^{\circ} \mathrm{C}$ for $2 \mathrm{~h}$. After $2 \mathrm{~h}$, sodium borohydride $0.6 \mathrm{M}(10 \mathrm{~mL})$ solution was added to the reflux solution dropwise, followed by the addition of a solution of trisodium citrate $0.1 \mathrm{M}(10 \mathrm{~mL})$ and refluxed for $2 \mathrm{hr}$ at $90^{\circ} \mathrm{C}$. The solution was centrifuged, and the supernatant was decanted away. The remaining solid was washed with copious amounts of hot water $\left(60^{\circ} \mathrm{C}\right)$ and ethanol. The solid was dried under vacuum at $40^{\circ} \mathrm{C}$.

\section{Synthesis of graphene decorated with nickel nanoparticles (Ni-rGO)}

Reduced graphene oxide-nickel nanoparticle composite was synthesized according to the modified literature method $[18,19]$. Graphene oxide $(0.4 \mathrm{~g})$ was mixed with deionized water $(25 \mathrm{~mL})$ and ultrasonicated until the graphene was well dispersed and formed a dark brown solution. The solution was transferred into $250 \mathrm{~mL}$ three-neck volumetric flask. $\mathrm{NiCl}_{2}(0.3 \mathrm{~g})$ was weighed into the solution and refluxed for $1 \mathrm{~h}$. After $1 \mathrm{~h}$, solutions of $0.1 \mathrm{M}$ sodium polyacrylate $(5 \mathrm{ml})$ and $0.2 \mathrm{M}$ sodium borohydride $(2 \mathrm{~mL})$ were added dropwise and refluxed for $2 \mathrm{~h}$ at $90^{\circ} \mathrm{C}$ under an argon atmosphere. The solution was centrifuged, and the supernatant was decanted away. The remaining solid was washed with copious amounts of hot water $\left(60^{\circ} \mathrm{C}\right)$ and ethanol. The solid was dried under vacuum at $40{ }^{\circ} \mathrm{C}$.

\section{Preparation of graphene decorated with cobalt ferrite nanoparticles (FeCo-rGO)}

Graphene-cobalt ferrite composite was synthesized according to the modified literature method $[18,20]$. The synthesized graphene oxide $(0.6 \mathrm{~g})$ was dissolved in deionized water $(40 \mathrm{~mL})$ in a $250 \mathrm{~mL}$ three-neck round-bottom flask by ultrasonication. The graphene oxide solution was then refluxed at $100{ }^{\circ} \mathrm{C}$ with $\mathrm{FeCl}_{3}(0.6 \mathrm{~g}), \mathrm{FeSO}_{4}(0.3 \mathrm{~g}), \mathrm{CoAc}_{2}$ $(0.8 \mathrm{~g})$, ammonium chloride $(0.3 \mathrm{~g})$ and $\mathrm{NaOH}(0.4 \mathrm{~g})$ under an argon atmosphere. The solution was refluxed for $1 \mathrm{~h}$ which ensured the metal salts were well dispersed. To the solution a solution of sodium borohydride $0.04 \mathrm{M}(10 \mathrm{~mL})$ was added dropwise and refluxed for an hour. The solution was cooled to room temperature and centrifuged, and the supernatant was decanted away. The remaining solid was 
Fig. 3 a SAED pattern of Ni-rGO, b SAED pattern of Co-rGO and $\mathbf{c}$ SAED pattern of FeCo-rGO
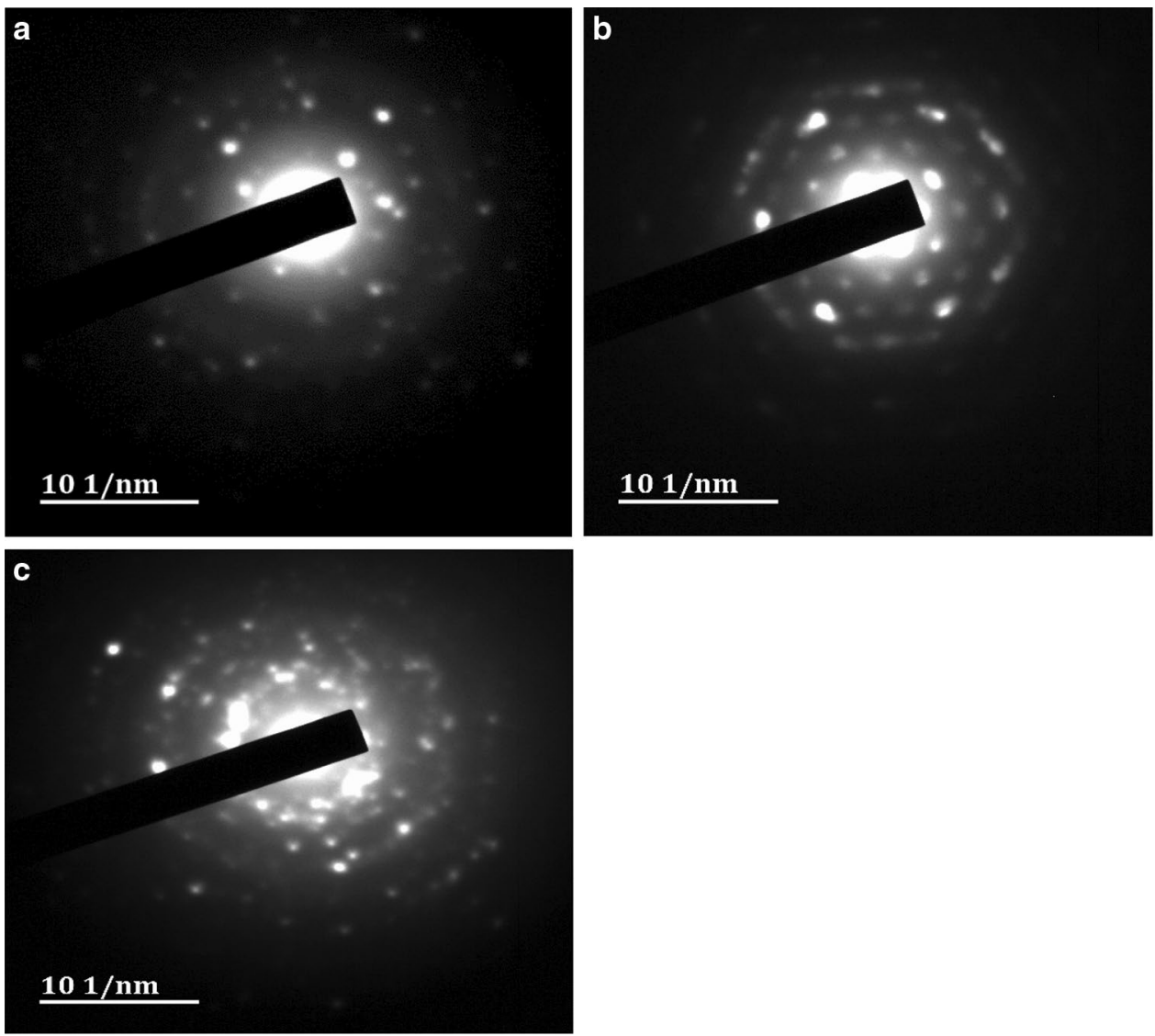

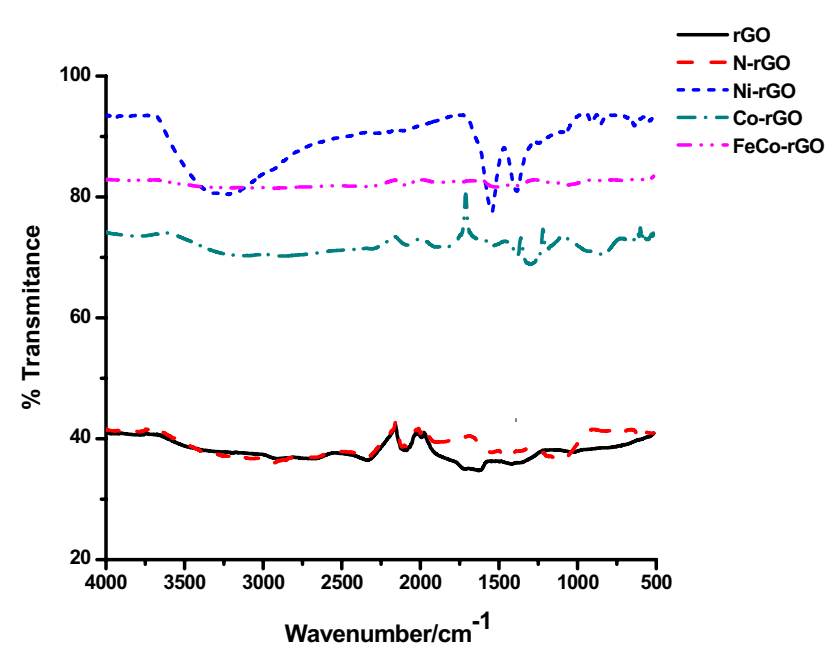

Fig. 4 FTIR spectra of rGO, N-rGO, Ni-rGO, Co-rGO and FeCorGO

washed with copious amounts of hot water, 1:1 $\mathrm{HCl}$ and ethanol. The solid was dried under vacuum at $40{ }^{\circ} \mathrm{C}$.

\section{Proof of principle (the adsorption of $\mathrm{Cr}$ (IV) from aqueous solution using $\mathrm{Co}-\mathrm{rGO}$ ).}

The adsorption experiment was conducted in a series of Erlenmeyer flasks containing $50 \mathrm{~mL}$ solution of $\mathrm{Cr}(\mathrm{VI})$ ion. The adsorption tests were conducted to evaluate the uptake of the $\mathrm{Cr}(\mathrm{IV})$ ion by Co-rGO. After this, the Co-rGO was separated from solution with the aid of rareearth magnet. The supernatant was collected, and the remaining heavy metal ion was evaluated using ICP-OES. The $\mathrm{Cr}(\mathrm{VI})$ solutions were fashioned from $1000 \mathrm{mg} / \mathrm{L}$ standard solution. A working solution of $100 \mathrm{mg} \mathrm{L}^{-1}$ was used to create samples ranging from 10 to $80 \mathrm{mg} \mathrm{L}^{-1}$.

Table 1 Table of elemental analysis

\begin{tabular}{lllll}
\hline Sample & C & N & H & O \\
\hline GO & 47.62 & 0.38 & 2.27 & 30.73 \\
rGO & 74.12 & 0.37 & 1.77 & 23.74 \\
N-rGO & 77.81 & 8.12 & 1.92 & 12.08 \\
\hline
\end{tabular}




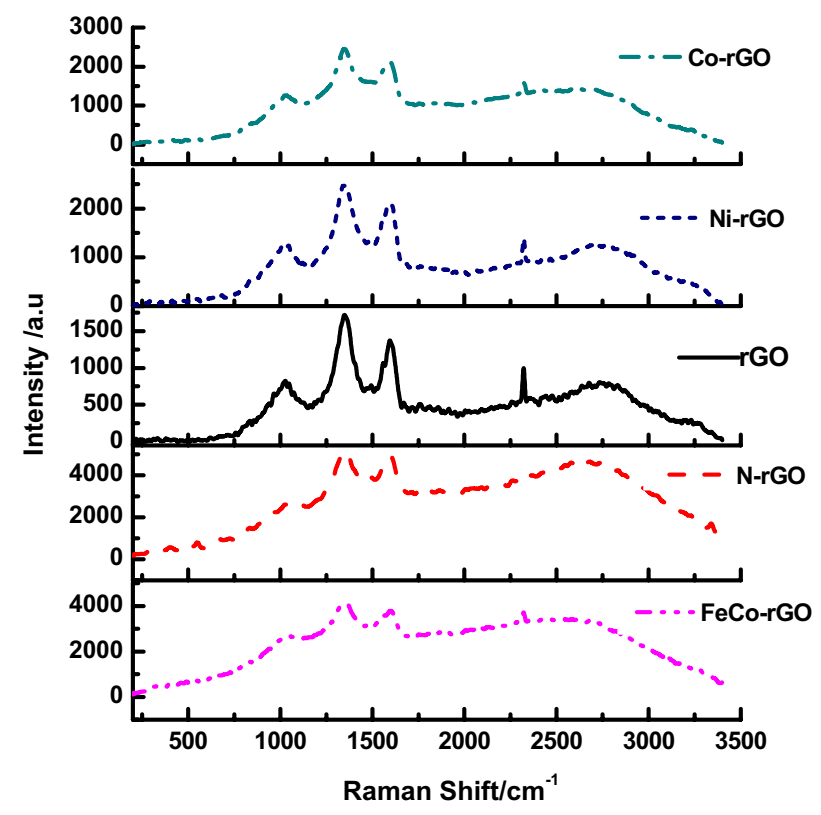

Fig. 5 Raman spectra of rGO, N-rGO, Ni-rGO, Co-rGO and FeCorGO
Table 2 Table of Raman spectroscopy data

\begin{tabular}{lllll}
\hline Sample & D band $/ \mathrm{cm}^{1}$ & $\mathrm{G}$ band $/ \mathrm{cm}^{1}$ & $2 \mathrm{D} / \mathrm{cm}^{1}$ & $\mathrm{I}_{\mathrm{D}} / \mathrm{I}_{\mathrm{G}}$ \\
\hline rGO & 1382 & 1580 & 2780 & 1.22 \\
N-rGO & 1360 & 1600 & 2680 & 1.12 \\
Ni-rGO & 1362 & 1600 & 2660 & 1.32 \\
Co-rGO & 1376 & 1580 & 2720 & 1.22 \\
FeCo-rGO & 1368 & 1590 & 2640 & 1.13 \\
\hline
\end{tabular}

The $\mathrm{pH}$ of the solution was adjusted to eight by adding drops of a concentrated sodium hydroxide solution. The removal efficiency $(R, \%)$ and the amount of heavy metal ions adsorbed $q(\mathrm{mg} / \mathrm{g})$ were determined according to the formula:

$R=\frac{\left(C_{0}-C_{t}\right)}{C_{0}} \times 100 \%$,

where $R$ is the removal efficiency of the metal ions, $C_{0}$ $\left(\mathrm{mg} \mathrm{L}^{-1}\right)$ is the initial concentration of the metal ion, and $C_{t}\left(\mathrm{mg} \mathrm{L}^{-1}\right)$ is the concentration of the metal ion at time $(t)$.
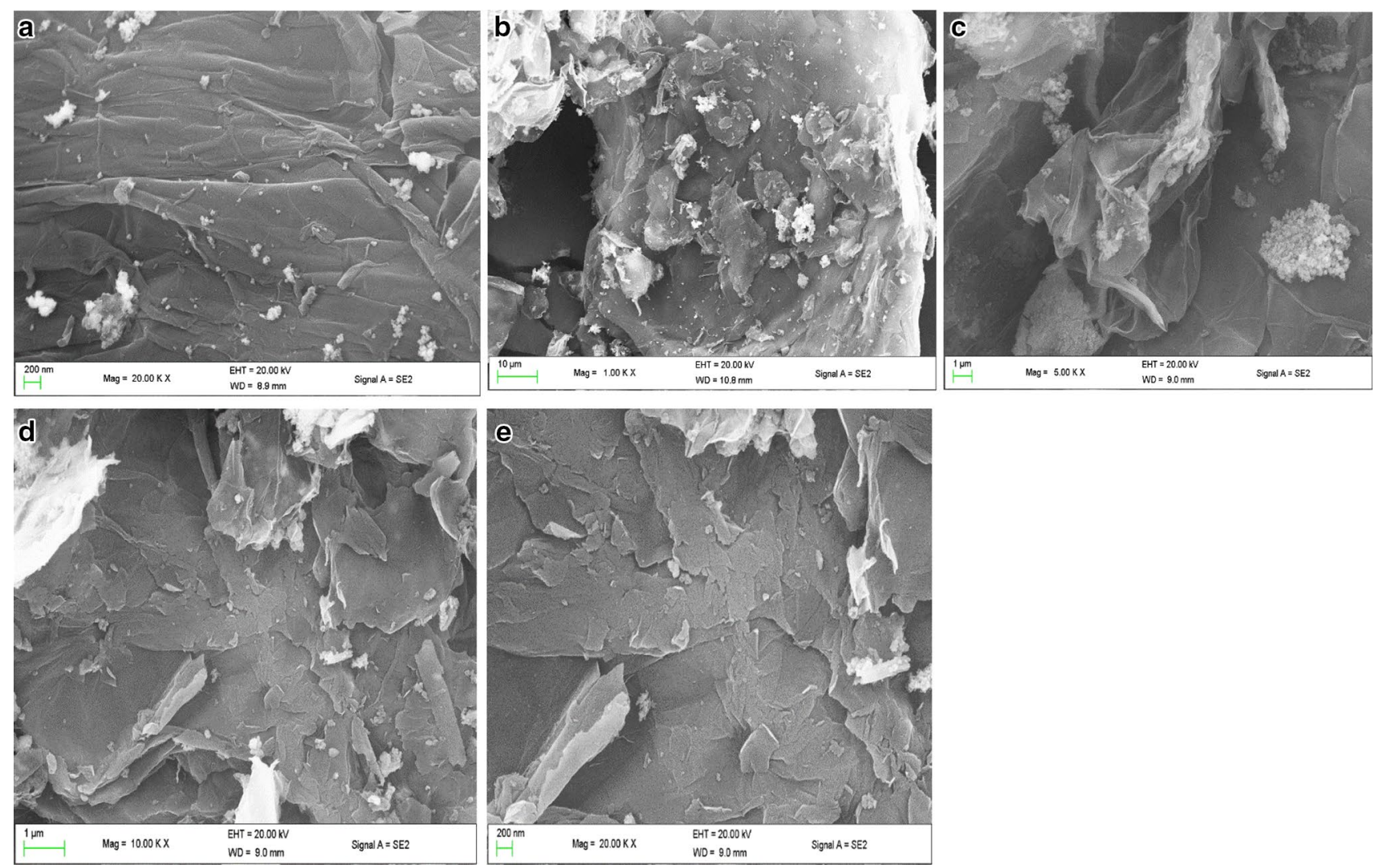

Fig. 6 SEM images of a Co-rGO, b Ni-rGO, c FeCo-rGO, d N-rGO and e rGO 
Table 3 Metal concentration as determined by ICP-OES for specimens

\begin{tabular}{lcccc}
\hline Specimen & \multicolumn{2}{l}{ Metal concentration/ppm } & \multirow{2}{*}{ Metal\% } \\
\cline { 2 - 4 } & Nickel & Iron & Cobalt & \\
\hline Ni-rGO & 121.23 & - & - & 15 \\
Co-rGO & - & - & 84.35 & 10 \\
FeCo-rGO & - & 59.52 & 30.15 & 7 \\
\hline
\end{tabular}

The adsorption experiment was done using concentrations of 20, 40, 6080 and $100 \mathrm{mg} \mathrm{L}^{-1}$, at the predetermined adsorbent dosage. The adsorption of $\mathrm{Cr}(\mathrm{VI})$ on $\mathrm{Co}-\mathrm{rGO}$, was evaluated by calculation of the removal efficiency. The regeneration of the Co-rGO was done by sonicating the $\mathrm{Co}-\mathrm{rGO}$ in ethanol.
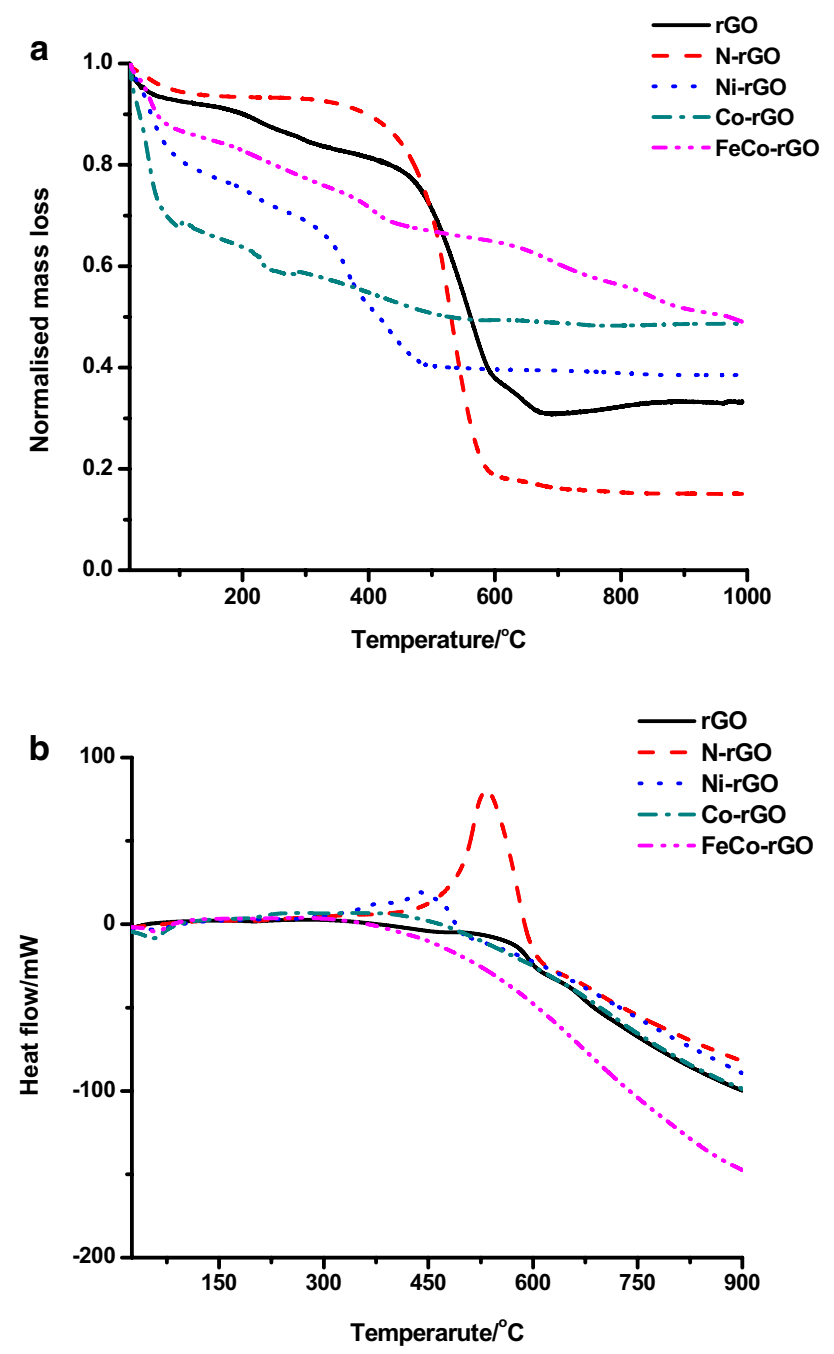

Fig. 7 Thermogram of the prepared samples a TGA and b DSC

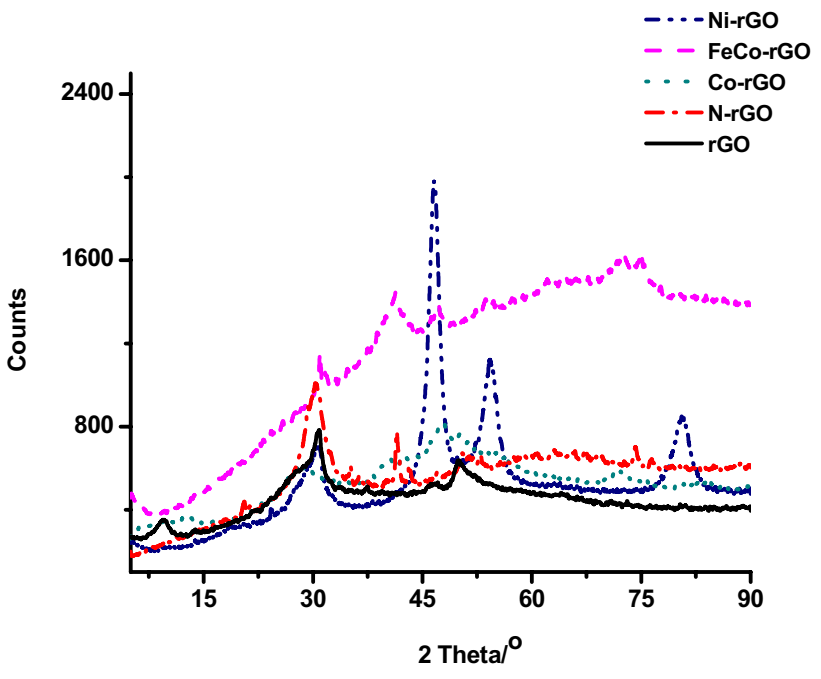

Fig. 8 XRD spectra of the specimens

\section{Results and discussion \\ Synthesis and characterization}

The synthesis of reduced graphene oxide decorated with magnetic nanoparticles was successful, as seen by inspection of TEM images in Fig. 1. TEM data show the prepared specimens: (a) Co-rGO, (b) Ni-rGO, (c) FeCo-rGO, (d) rGO, (e) N-rGO. The cobalt nanoparticles were monodispersed in the graphene sheet. Ni-rGO specimen had nanoparticles that were randomly dispersed and were larger than those found in the Co-rGO specimen. The FeCo-rGO had nanoparticles which were slightly agglomerated. TEM was used to determine the particle size distribution as shown in Fig. 2 using the Image $\mathbf{J}$ software. The average particle size along with the standard deviation was also determined. The total number of particles for the analysis of particle size determination was 50 particles per sample. It was found that the particle size distribution for Co-rGO was determined to be between 1.32 and $3.25 \mathrm{~nm}$ and the average particle size was 1.89 with a standard deviation of 0.531 . Ni-rGO had a particle size distribution between 11.23 and $47 \mathrm{~nm}$, and the average particle size was $35.12 \mathrm{~nm}$ with the standard deviation of 10.45. Ni-rGO had a particle size distribution between 10.23 and $32.15 \mathrm{~nm}$, and the average particle size was $18.12 \mathrm{~nm}$ with the standard deviation of 7.34 . It has been widely reported in literature that the nanoparticle size and shape stem from the way they were produced. The most common factors are the nature of the stabilizing agent and its concentration [21]. In the production of silver nanoparticles, monodentate stabilizing agent such as PVP leads 
Table 4 Magnetization data for the prepared specimens

\begin{tabular}{lllll}
\hline Specimen & $\begin{array}{l}\text { Saturation magnetiza- } \\
\text { tion/(emu/g) }\end{array}$ & Coercivity/(Oe) & $\begin{array}{l}\text { Remanence magnetiza- } \\
\text { tion/(emu/g) }\end{array}$ & $\begin{array}{l}\text { Magnetic } \\
\text { susceptibil- } \\
\text { ity }\end{array}$ \\
\hline rGO & 1.34 & 299 & 1.36 & $8.9 \times 10-5$ \\
N-rGO & 1.40 & 299 & $1.39112 .54 / 1$ & $4.6 \times 10^{-4}$ \\
Ni-rGO & 3.64 & 256 & 3.54 & $2.4 \times 10^{-4}$ \\
Co-rGO & 6.82 & 266 & 6.95 & $4.5 \times 10^{-4}$ \\
FeCo-rGO & 12.54 & 237 & 12.52 & $8.4 \times 10^{-4}$ \\
Ni-NP & 54.93 & 48 & 55.25 & $3.9 \times 10^{-3}$ \\
Co-NP & 20.12 & 67 & 20.09 & $1.5 \times 10^{-3}$ \\
FeCoO-NP & 47.28 & 188 & 47.08 & $3.4 \times 10^{-3}$ \\
\hline
\end{tabular}

to cube-shaped nanoparticles and multidentate stabilizing agent such as citrate leads to diamond-shaped nanoparticles [21]. The selected area electron diffraction (SAED) is shown in Fig. 3a-c. SAED was done for the Co-rGO, Ni-rGO and FeCo-rGO samples. The spectra have spots which show that the samples were monocrystalline samples [22]. The nanoparticles are colloidal due to the manner in which they were produced. Infrared spectra with characteristic absorption frequencies of the specimens are shown in Fig. 4. The stretching frequency of phenolic and carboxylic $\mathrm{O}-\mathrm{H}$ appears at $\sim 3405 \mathrm{~cm}^{-1}$ [23]. The stretching frequency of $\mathrm{C}=\mathrm{O}$ appears both at $\sim 1559 \mathrm{~cm}^{-1}$ and $\sim 1381 \mathrm{~cm}^{-1}$ [24]. The $\mathrm{C}=\mathrm{C}$ vibration frequency of benzene rings occurs at $\sim 681 \mathrm{~cm}^{-1}$ [23]. The doped reduced graphene oxide showed the characteristic peak found in grapheme, indicating the introduction of dopant such as: $\mathrm{O}-\mathrm{H}\left(3240 \mathrm{~cm}^{-1}\right), \mathrm{C}=\mathrm{C}\left(1725 \mathrm{~cm}^{-1}\right)$, and $\mathrm{C}=\mathrm{N}\left(1570 \mathrm{~cm}^{-1}\right)$ [25]. The rGO and nitrogen-doped samples were also analyzed using elemental analysis. The data was summarized in Table 1 , and it was found that the nitrogen-doped reduced graphene oxide was found to be $8 \%$ nitrogen.

Raman spectra are shown in Fig. 5 and summarized in Table 2. The D-band, which is located around $1360 \mathrm{~cm}^{-1}$, is used to determinate the level of disorder, imperfection and impurities in graphene, while the G-band corresponds to the vibration of $s p^{2}$ hybridized carbon-bonded atoms in the graphene lattice [26]. The ratio of $I_{D} / I_{G}$ in both the spectra was greater than 1 showing the defects and disorder in the graphene structure, which is common in reduced graphene oxide that is multilayered. The Raman data are complemented by the SEM images as shown in Fig. 6. The SEM data show that the samples had a wrinkled morphology and were multilayered. This complements the Raman data where the samples have a broad 2D band at $2700 \mathrm{~cm}^{-1}$ [27]. The SEM data for the Co-rGO, Ni-rGO and FeCo-rGO show the presence of nanoparticles on the graphene flakes, complementing the SAED spectra and showing that the samples are monocrystalline and the metal nanoparticles are on the composite samples. The SEM, TEM and Raman results showed that the rGO was not single layer pristine sheets. To further determine the metal loading, the composite samples were analyzed using ICP-OES. The ICP-OES data are summarized in Table 3. The metal loading in each specimen was $10 \%$ (w/w) cobalt for $\mathrm{CoG}, 11 \%$ (w/w) nickel for $\mathrm{NiG}$ and $15 \%$ (w/w) iron and 7\% cobalt for FeCo-rGO.

The thermal characteristics of the specimens were analyzed using TGA and DSC. The thermograms shown in Fig. 7 show the degradation patterns from room temperature to $1000{ }^{\circ} \mathrm{C}$. TGA thermograms in Fig. 7a showed that the specimens had a multistep degradation pattern. rGO, Co-rGO and $\mathrm{Ni}-\mathrm{rGO}$ specimens had three major mass loss degradations. The rGO specimen had the following mass loss pattern: room temperature to $150{ }^{\circ} \mathrm{C}$ there was a mass loss of $10 \%$ due to water loss or any absorbed moisture and gases; $200-400{ }^{\circ} \mathrm{C}$ there was a mass loss of $15 \%$, which was due to the destruction of loosely bound oxygen-containing compounds; $400-670{ }^{\circ} \mathrm{C}$ there was a mass loss of $45 \%$, which was due to the destruction of graphene nanosheets [24, 28]. Co-rGO specimen had the following degradation patterns: room temperature to $100{ }^{\circ} \mathrm{C}$ there was a mass loss of $20 \%$, which was due to moisture loss or adsorbed gases; $150-300{ }^{\circ} \mathrm{C}$ there was a mass loss of $10 \%$, which was due to the degradation of labile oxygen-containing compounds; $10 \%$ mass loss was due to the degradation of graphene nanosheets that occurred between 300 and $800{ }^{\circ} \mathrm{C}$. Ni-rGO had the following mass loss degradation pattern: the first was from room temperature to $100{ }^{\circ} \mathrm{C}$ with a mass loss of $25 \%$ due to water loss; the second degradation was a mass loss of $25 \%$, from 200 to $320^{\circ} \mathrm{C}$ which was due to the destruction of loosely bound oxygen-containing compounds; and a mass loss of $25 \%$ from 330 to $750{ }^{\circ} \mathrm{C}$ was due to the degradation of graphene nanosheets. FeCo-rGO had multistep degradation in three steps: from room temperature to $100{ }^{\circ} \mathrm{C}$ there was a mass loss of $12 \%$, due to moisture loss; $120-450{ }^{\circ} \mathrm{C}$ there was a mass loss of $20 \%$, due to the degradation of loosely bound oxygen-containing compounds; $450-750{ }^{\circ} \mathrm{C}$ there was a mass loss of $25 \%$ due to the destruction of graphene 


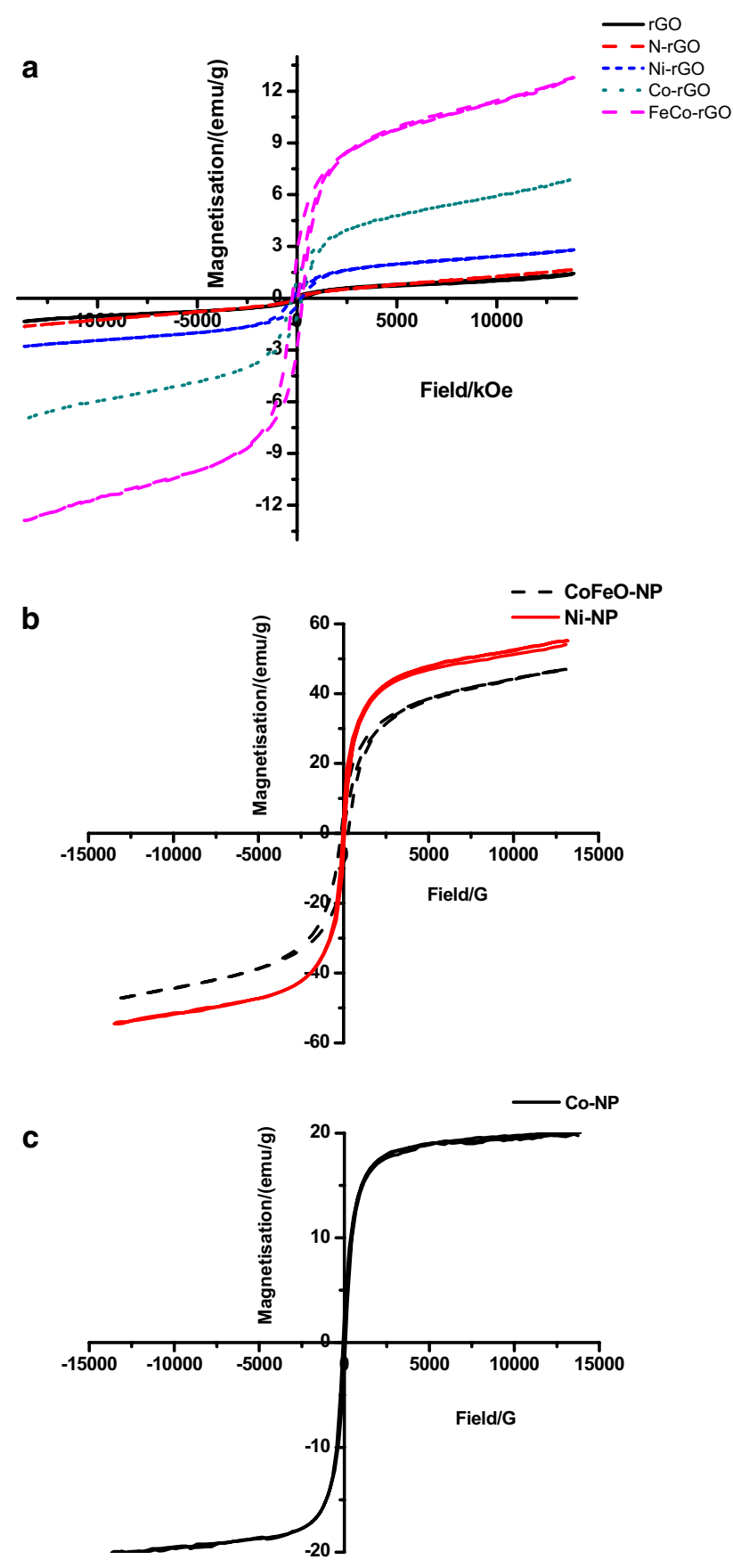

Fig. 9 VSM spectrum of the prepared samples

nanosheets $[24,28,29]$. The $\mathrm{N}-\mathrm{rGO}$ sample only had two step degradation with moisture loss. A weight loss of $80 \%$ from $400{ }^{\circ} \mathrm{C}$ to $700{ }^{\circ} \mathrm{C}$ indicates the co-decomposition of nitrogen and graphene [30, 31].

The DSC data in Fig. $7 \mathrm{~b}$ shows the endothermic and exothermic process of the specimens. The Ni-rGO specimen had a pronounced degradation peak at $450{ }^{\circ} \mathrm{C}$, due to the degradation of graphene nanosheets [29]. N-rGO was the only sample which had a prominent peak due to the endothermic degradation of the nitrogen-doped reduced graphene oxide sheets. The doping marginally improved the thermal stability of the rGO. The rGO sample also had a slight bump at $580{ }^{\circ} \mathrm{C}$, corresponding to the destruction of graphene nanosheets. $\mathrm{Co}-\mathrm{rGO}$ and $\mathrm{CoFe}-\mathrm{rGO}$ specimens had no observed peaks.

The XRD in Fig. 8 shows the diffraction of spectra for all composite specimens. The composite specimens were compared to the spectrum of specimen $\mathrm{rGO}$, which had prominent peaks at $26^{\circ}$ and $49^{\circ}$ [31]. All the composite specimens had characteristic rGO peaks at $26^{\circ}$, which indicate all the samples are reduced graphene oxide based. $\mathrm{Ni}-\mathrm{rGO}$ had the diffraction peaks at $26^{\circ}, 45^{\circ}, 55^{\circ}$ and $80^{\circ}$. The peaks at 26 correspond to the rGO, while peaks at $45^{\circ}, 55^{\circ}$ and $80^{\circ}$ correspond to nickel nanoparticles [32, 33]. The FeCo-rGO specimen had a peak at $26^{\circ}, 50^{\circ}$ and $72^{\circ}$ [34]. Co-rGO had a peak at $26^{\circ}$ due to graphene, $47^{\circ}$ due to cobalt nanoparticle [35] and 76 which was unreported. The average particle size for the nanoparticles was calculated using the Scherrer equation [27], $\tau=\frac{k \lambda}{\beta \cos \theta}$, where $\tau$ is the particle size, $\beta$ is the full width at half maximum and $k$, is a dimensionless shape factor. The cobalt nanoparticles were $8 \mathrm{~nm}$, the nickel nanoparticles were $15 \mathrm{~nm}$ and FeCo nanoparticles were $18 \mathrm{~nm}$.

\section{Magnetic properties}

The magnetic response of the specimens was determined by the vibrating sample magnetometer (VSM). The specimens were vibrated in the presence of a sweeping electromagnetic field from - 15 to $15 \mathrm{kOe}$ and recorded in Table 4 . The rGO exhibits some magnetization which has been also been observed in literature $[14,15,36]$. It has been theorized that the zigzag pattern on the armchair model of the graphene introduces localized metallicity on the graphene. The data obtained from the VSM results show that rGO had magnetization of $1.36 \mathrm{emu}^{-1}$ The nitrogen doping sample had slightly higher magnetization than the rGO. Here, the nitrogen has the same atomic radius as carbon, and it can be easily assimilated into the graphene matrix. The nitrogen doping increases the spin population, which increases the magnetization of the doped sample [14, 15, 37]. Graphene by itself has been reported from theoretical calculations to have long-range ferromagnetic ordering resulting from the point defects [38]. Doping graphene has been shown to introduce paramagnetic properties into the grapheme, resulting from the dopants neutralizing the point defects that induce ferromagnetism in graphene [38]. The composite samples were found to exhibit superparamagnetic behavior, which is characterized by small coercivity. This was expected because the nanoparticles decorating reduced graphene oxide were magnetic [32-35]. The hysteresis loops of $\mathrm{FeCo}-\mathrm{rGO}$ and $\mathrm{Co}-\mathrm{rGO}$ specimens, shown 
Fig. 10 Diagram showing the attraction of the samples using a magnet, $\mathbf{a}$ Co-rGO and $\mathbf{b}$ rGO
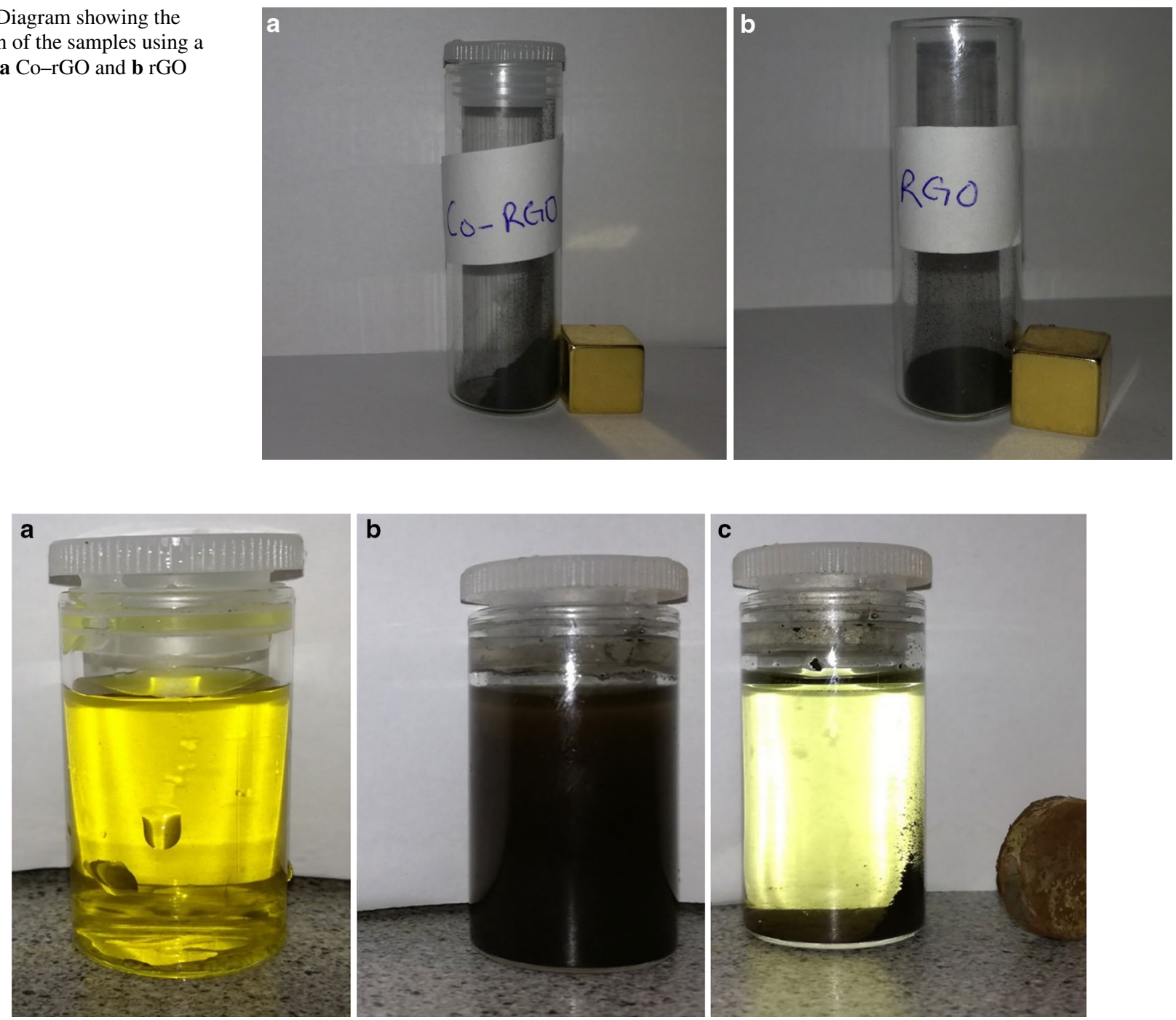

Fig. 11 Diagram showing the adsorption of $\mathrm{Cr}(\mathrm{VI})$ using $\mathrm{Co}-\mathrm{rGO}$ and the separation using $\mathrm{Co}-\mathrm{rGO}$ using a magnet

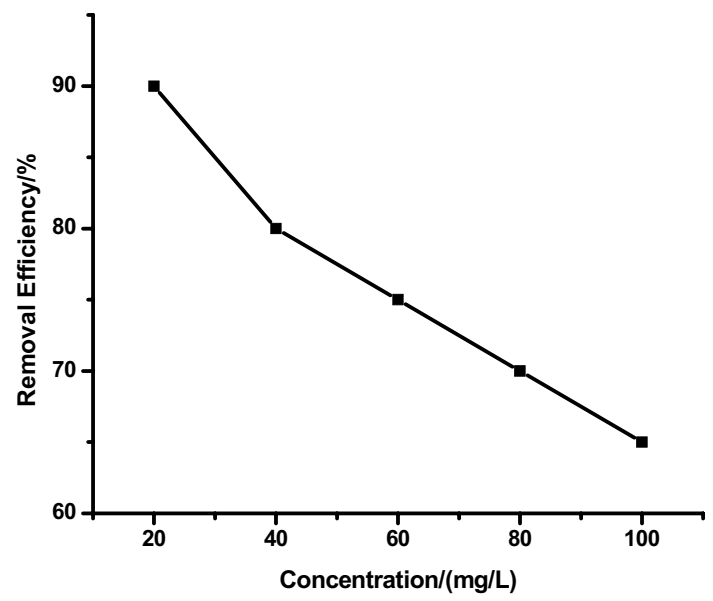

Fig. 12 Removal efficiency of the $\mathrm{Cr}(\mathrm{VI})$ using Co-rGO

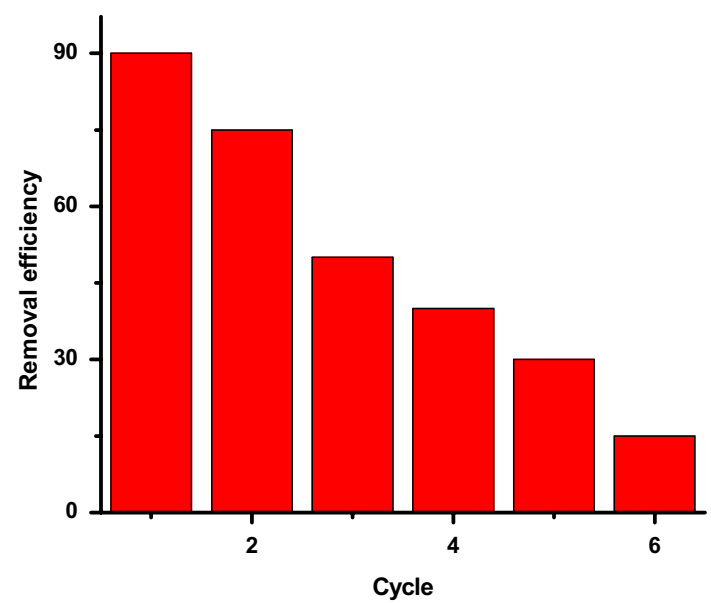

Fig. 13 The removal efficiency of recycled CO-rGO 


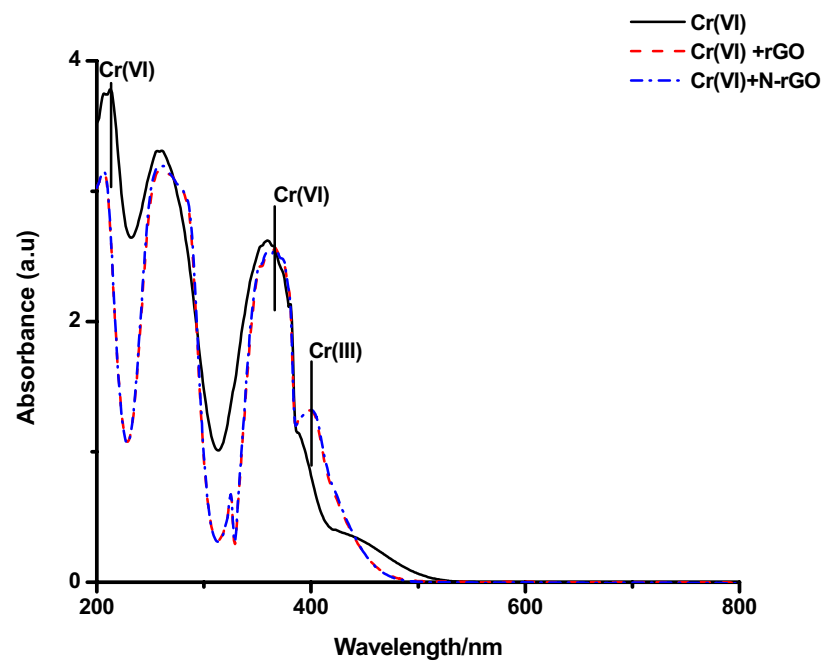

Fig. 14 Plot of UV/Vis spectrum of 100 ppm Cr(VI) standard, Cr(VI) after adding $\mathrm{N}-\mathrm{rGO}$ and $\mathrm{rGO}$

in Fig. 9, do not show saturation due to limited applied field. At an applied field of $15 \mathrm{kOe}$, the specimen magnetization response of $\mathrm{FeCo}-\mathrm{rGO}$ was $12.5 \mathrm{emu} \mathrm{g}^{-1}$ and that of Co-rGO was $6.8 \mathrm{emu} \mathrm{g}^{-1}$. Ni-rGO and rGO started to saturate at $3.6 \mathrm{emus}^{-1}$ and $1.3 \mathrm{emu} \mathrm{g}^{-1}$, respectively. All the specimens have low coercivity suggesting that they are soft magnetic materials [4]. The composite samples exhibit superparamagnetic behavior due to a charge transfer from the nanoparticle to the reduced graphene oxide, which occurs from the interfacial electronic interaction between the reduced graphene oxide (rGO) and the metallic nanoparticles [9]. The charge transfer between graphene and metallic nanoparticles changes the electronic and magnetic properties in the metal graphene composite. Figure 10 shows that the samples were attracted by magnet, except for rGO. The nanoparticles were also tested using the VSM. It was shown that the nickel nanoparticles and the cobalt nanoparticles had a low coercivity. The formation of the graphene nanocomposite decreased the saturation magnetization. The graphene quenched the magnetization. It has also been reported that the small nanoparticles such as cobalt nanoparticles usually exhibit superparamagnetic behavior, while the larger particle start exhibiting a slight ferromagnetic behavior, which was studied using different sizes of nickel nanoparticles [39]. The electronic interaction between the nanoparticles and the rGO changes the magnetic anisotropy, which in turn increases the coercivity and loss of superparamagnetism to ferromagnetic behavior [39]. The Parameters such as defect in the structure and the orientation increase the coercivity and decrease the magnetization [39]. All the samples can be applied in water purification to adsorb and remove the pollutants. The sample with the adsorbed pollutant can be removed using a handheld magnet. The ideal samples to be used in the removal of pollutants were $\mathrm{Ni}-\mathrm{rGO}, \mathrm{Co}-\mathrm{rGO}, \mathrm{N}-\mathrm{rGO}$ and $\mathrm{FeCo}-\mathrm{rGO}$. An example of water purification using graphene nanoparticle composite is highlighted below using Co-rGO in the removal of $\mathrm{Cr}(\mathrm{VI})$ in water.

\section{Adsorption experiments}

The adsorption of $\mathrm{Cr}$ (VI) was done using $20 \mathrm{mg}$ of Co-rGO and agitating the mixture for $3 \mathrm{~h}$. The concentrations tested were between 80 and $20 \mathrm{mg} \mathrm{L}^{-1}$. The concentration of $20 \mathrm{mg} \mathrm{L}^{-1}$ had the highest removal efficiency as shown in Fig. 11. Figure 12 shows the adsorption of $\mathrm{Cr}(\mathrm{VI})$, where (a) is the a solution of the $\mathrm{Cr}(\mathrm{VI})$ by itself, (b) is the mixture $\mathrm{Cr}(\mathrm{VI})$ and $\mathrm{Co}-\mathrm{rGO}$, and (c) the separation of $\mathrm{Cr}(\mathrm{VI})$ adsorbed on Co-rGO. The Co-rGO was recycled using ethanol which was sonicated and removed by a handheld magnet. The Co-rGO was then dried in a vacuum oven at $60{ }^{\circ} \mathrm{C}$. The concentration used for regeneration of $\mathrm{Co}-\mathrm{rGO}$ was $20 \mathrm{mg} \mathrm{L}^{-1}$. The regeneration of Co-rGO was achieved by ultrasonicating the composite in ethanol. Figure 13 shows the graphs of the number of regeneration with the corresponding removal efficiency. It ultimaly shows the decrease in perfomance of the Co-rGO in removing $\mathrm{Cr}(\mathrm{VI})$ ions with each cylcle. The drop in the removal efficiency between the first and the fifth regeneration of Co-rGO was from 75 to $15 \mathrm{mg} \mathrm{L}^{-1}$. After the fifth regeneration of Co-rGO, the efficiency dropped significantly, showing that $\mathrm{Co}-\mathrm{rGO}$ can be re-used five times to have the maximum removal of $\mathrm{Cr}(\mathrm{VI})$ as possible.

The other synthesized samples such as $\mathrm{N}-\mathrm{rGO}$ and rGO were also tested using a $100 \mathrm{ppm}$ standard of $\mathrm{Cr}(\mathrm{VI})$. A mass of $30 \mathrm{mg}$ was added to $50 \mathrm{~mL}$ of $100 \mathrm{ppm}$ standard and agitated for $3 \mathrm{~h}$. There was no observable removal of $\mathrm{Cr}(\mathrm{VI})$ ions from solution when using ICP analysis. The solution was then analyzed using UV/Vis spectrophotometry as shown in Fig. 14. The UV/Vis spectrum revealed that the sample $\mathrm{N}-\mathrm{rGO}$ and rGO had reduced the $\mathrm{Cr}(\mathrm{VI})$ ions to $\mathrm{Cr}(\mathrm{III})$. This is evident in the appearance of the $\mathrm{Cr}$ (III) absorption peak at $\lambda_{\max } 398 \mathrm{~nm}$. It has been observed in literature that the positively charged rGO function groups tend to facilitate the reduction of the $\mathrm{Cr}(\mathrm{VI})$ to $\mathrm{Cr}(\mathrm{III})$ [40]. $\mathrm{Cr}(\mathrm{VI})$ ions in the form of $\mathrm{HCrO}_{4}{ }^{-}$which is attracted to the positively charged centers of the rGO.

\section{Conclusion}

Magnetic reduced graphene oxide specimens were synthesized with varying degrees of magnetic behavior. Nitrogen-doped graphene showed that the nitrogen introduced thermal stability in graphene. Magnetization experiments showed that nitrogen increases the saturation magnetization 
of the reduced graphene oxide and decreases coercivity. The doping of graphene with nitrogen changes the magnetic property of graphene. The anchoring of magnetic nanoparticles on reduced graphene oxide was successfully completed from a modified method in literature. The nanoparticles (cobalt, cobalt ferrite and nickel) were monodispersed on the graphene. The composites were found to be less thermally stable when compared to graphene. All samples were magnetic and $\mathrm{FeCo}-\mathrm{rGO}$ had the highest saturation magnetization at $12.5 \mathrm{emu} \mathrm{g}^{-1}$. The samples Ni-rGO, Co-rGO, and FeCo-rGO were easily attracted by a magnet. Thus, they can be further applied in the adsorption of toxic metal and organic pollutants in water. $\mathrm{Co}-\mathrm{rGO}$ was used as proof of principle in water purification and it was found that it can be reused five times. The other samples synthesized catalysed the reduction of $\mathrm{Cr}(\mathrm{VI})$ to $\mathrm{Cr}(\mathrm{III})$.

Acknowledgements Funding was received from South African Agency for Science and Technology Advancement with Grant No. 86042459.

Open Access This article is licensed under a Creative Commons Attribution 4.0 International License, which permits use, sharing, adaptation, distribution and reproduction in any medium or format, as long as you give appropriate credit to the original author(s) and the source, provide a link to the Creative Commons licence, and indicate if changes were made. The images or other third party material in this article are included in the article's Creative Commons licence, unless indicated otherwise in a credit line to the material. If material is not included in the article's Creative Commons licence and your intended use is not permitted by statutory regulation or exceeds the permitted use, you will need to obtain permission directly from the copyright holder. To view a copy of this licence, visit http://creativecommons.org/licenses/by/4.0/.

\section{References}

1. Akbarzadeh, A., Samiei, M., Soodabeh, D.: Magnetic nanoparticles: preparation, physical, and applications in biomedicine. Nanoscale. Res. Lett. 7, 144-156 (2012)

2. Song, Q., Zhang, Z.J.: Shape control and associated magnetic properties of spinel cobalt ferrite nanocrystals. J. Am. Chem. Soc. 126, 6164-6468 (2004)

3. Cruz, J.C., Nascimento, M.A., Amaral H.A.V., Lima, D.S.D., Teixeria, A.P.C., Lopes, R.P.: Synthesis and characterization of cobalt nanoparticles for application in the removal of textile dye. J. Environ. Manage. 244, 220-228 (2019)

4. Mekuria, S.L., Addisu, K.D., Chou, H.-Y., Hilemeskel, B.Z., Tsai, H.-C.: Potential fluorescence and magnetic resonance imaging modality using mixed lanthanide oxide nanoparticles. Colloids. Surf. B. 167, 54-62 (2018)

5. Dey, C., Baishya, K., Ghosh, A., Goswami, M.M., Ghosh, A., Mandal, K.: Improvement of drug delivery by hyperthermia treatment using magnetic cubic cobalt ferrite nanoparticles. J. Magn. Magn. Mater. 427, 168-174 (2017)

6. Yin, K., Ji, J., Shen, Y., Xiong, Y., Bi, H., Sun, J. Xu, T., Zhu, Z., Sun, L.: Magnetic properties of $\mathrm{Co}_{3} \mathrm{O}_{4}$ nanoparticles on graphene substrate. J. Alloys. Compd. 720, 345-351 (2017)

7. Wong, D., Zhou, J., Li, J., Jiang, X., Wang, Y., Gao, F.: Cobaltboron nanoparticles anchored of graphene as anode of lithium ion batteries. Chem. Eng. J. 360, 271-279 (2019)
8. Shao, J., Feng, J., Zhou, H., Yuan, A.: Graphene aerogel encapsulated $\mathrm{Fe}-\mathrm{Co}$ oxide nanocubes derived from Prussian blue analogue as integrated anode with enhanced Li-ion storage properties. Appl. Surf. Sci. 471, 745-752 (2019)

9. Meng, F., Wang, H., Huang, F., Guo, Y., Z. Wang, Hui, D.: Graphene-based microwave absorbing: a review and prospective. Compos. B. 137, 260-277 (2018)

10. Taghavi, S., Tavasoli, A., Asghari, A., Signoretto, M.: Loading and promoter effects on the performance of nitrogen functionalized graphene nanosheets supported cobalt Fischer-Tropsch synthesis catalysts. Int. J. Hydrog. Energy. 44, 10604-10615 (2019)

11. Zhang, Y., Tang. Z-R., Xu, F., Xu, Y-J, Xu.: TiO2-Graphene nanocomposites for gas phase photocatalytic degradation of volatile aromatic pollutants is $\mathrm{TiO} 2-$ graphene truly different from other TiO2-carbon composite? ACS Nano. 4, 7,303-7314 (2014)

12. Williams, R., Sege, B., Kamat, P.V.: TiO2-graphene nanocomposites, UV assisted photocatalytic reduction of graphene oxide. ACS Nano 2, 1487-1491 (2008)

13. Khalil, I., Julkapli, N.M., Yehye, W.A., Basirum, W.J., Bhargava, S.K.: Graphene-gold nanoparticle hybrid-synthesis, functionalization, and application in a electrochemical and surface-enhanced Raman scattering biosensor. Materials. 9, 406-443 (2016)

14. Rao, C.N.R., Matte, H.S.S.R., Subrahmanyam, K.S., Maitra, U.: Unusual magnetic properties of graphene and related materials. Chem. Sci. 3, 45-52 (2012)

15. Rao, C.N.R., Gopalakrishnan, K., Govindaraj, A.: Synthesis, properties and application of Graphene doped with boron, nitrogen and other elements. Nano Today. 9, 324-343 (2014)

16. Marcano, D.C., Kosynkin, D.V., Berlin, J.M., Stinitsii, A.S., Sun, Z., Slesarev, A., Alemany, L.B., Lu, W., Tour, J.M.: Improved Synthesis of graphene oxide. ACS Nano. 4, 4806-4812 (2010)

17. Lui, X., Yu, Y., Niu, Y., Boa, S., Hu, W.: Cobalt nanoparticle decorated graphene aerogel for efficient oxygen reduction reaction electrocatalysis. Int. J. Hydrog. Energy. 24, 5930-5937 (2017)

18. Muszynski, R., Seger, B., Kamat, P.V.: Decorating graphene sheets with gold nanoparticles. J. Phys. Chem. C. Lett. 112, 5263-5266 (2008)

19. Barakat, N.A.M., Motlack, M., Ghouri, Z.K., Yasin, A.S., ElNewehy, M.H., Al-Deyab, S.S.: Nickel nanoparticle-decorated graphene as highly effective and stable electrocatalyst for urea electrooxidation. J. Mol. Catal. Chem. 421, 83-91 (2016)

20. Maaz, K., Mumtaz, A., Hasanain, S.K., Ceylan, A.: Synthesis and magnetic properties of cobalt ferrite $\left(\mathrm{CoFe}_{2} \mathrm{O}_{4}\right)$ nanoparticles prepared by wet chemical route. J. Magn. Magn. Mater. 308, 289-295 (2007)

21. Xia, Y., Xia, X., Peng, H.-C.: Shape-Control-Synthesis of colloidal metal nanocrystals: Thermodynamic versus kinetic method. J. Am. Chem. Soc. 137, 7947-7969 (2015)

22. Wu, H., Shao, M., Gu, J., Wei, X.: Microwave-assisted synthesis of fibre-like $\mathrm{Mg}(\mathrm{OH})_{2}$ nanoparticles in aqueous solution at room temperature. Mater. Lett. 58, 2166-2169 (2004)

23. Yang, H.T., Sum, Y.K., Shen, C.M., Yang, T.Z., Gao, H.J.: Synthesis and magnetic properties of $\varepsilon$-cobalt nanoparticles. Surf. Interface. Anal. 36, 155-162 (2004)

24. Gonsalves, I.R., Verenkar, V.M.S.: Synthesis and characterization of nanosized nickel-cobalt ferrite obtained by precursor combustion method. J. Meth. Anal. Calorim. 108, 877-903 (2012)

25. Xue, Y., Yu, D., Dai, L., Wang, R., Li, D., Roy, A., Lu, F., Chen, H., Lui, Y., Qu, J.: Three-dimensional B, N-doped graphene foam as a metal-free catalyst for oxygen reduction reaction. Phys. Chem. Chem. Phys. 15, 12220-12227 (2013)

26. Kim, S-C., Kim, B.H., Kim, S-J., Lee, Y-S. Kim, H-G., Lee, H., Park, S.H., Jung, S-C.: Preparation and characterization of cobalt/graphene composites using liquid phase plasma system. J. Nanosci. Nanotechnol.15, 228-231 (2015) 
27. Iqbal, M.W., Singh, A.K., Iqbal, M.Z., Eon, J.: Raman fingerprint of doping due to metal adsorbates on graphene. J. Phys. Condens. Matter. 24, 335301-335307 (2012)

28. Kumar, P.R., Kollu, P., Santhosh, C., Rao, K.E.V., Kim, D.K., Grace, A.N.: Enhanced properties of porous $\mathrm{CoFe}_{2} \mathrm{O}_{4}$-reduced graphene oxide composites with alginate binders for Li-ion battery application. N. J. Chem. 38, 3654-3661 (2014)

29. Chang, Y., Li, J., Wang, B., Lou, H., Zhi, L.: A facile synthetic approach to reduced graphene oxide- $\mathrm{Fe}_{3} \mathrm{O}_{4}$ composite as highperformance anode for lithium-ion batteries. J. Mat. Sci. Technol. 30, 759-764 (2014)

30. Wang, M., Huang, Y., Chen, X., Wang, K., Wu, H., Zhang, N., $\mathrm{Fu}, \mathrm{H}$.: Synthesis of nitrogen and sulfur co-doped graphene supported hollow $\mathrm{ZnFe}_{2} \mathrm{O}_{4}$ nanosphere composites for application in lithium-ion batteries. J. Alloy. Compd. 691, 407-415 (2017)

31. Suhas, D.P., Aminabhavi, T.M., Jeong, H.M., Raghu, A.V.: Hydrogen peroxide treated graphene as an effective nanosheet filler for separation application. RSC Adv. 5, 100984-100995 (2015)

32. Fu, C., Zhao, G., Zhang, H., Li, S.: Evaluation and Characterization of reduced graphene oxide nanosheets as anode material for lithium-ion batteries". Int. J. Electrochem. Sci. 8, 6269-6280 (2013)

33. Pawar, S.P., Stephen, S., Bose, S., Mittal, V.: Tailored electrical conductivity, electromagnetic and thermal transport in polymeric blends with graphene sheets decorated with nickel nanoparticles. Phys. Chem. Chem. Phys. 17, 14922-14930 (2015)
34. Fu, Y., Chen, H., Sun, X., Wang, X.: Combination of cobalt ferrite and graphene: high-performance and recyclable visible-light photocatalysis. Appl. Catal. B: Environ. 111-112, 280-287 (2012)

35. Long, Q., Xu, Z., Xiao, H., Xie, K.: A facile synthesis of a cobalt nanoparticle-graphene nanocomposite with high-performance and triple-band electromagnetic wave absorption properties. RSC Adv. 8, 1210-1217 (2018)

36. Katznelson, M.I.: Graphene: carbon in two dimensions. Mater. Today. 10, 20-27 (2007)

37. Zhu, J., Park, H., Podila, R., Wadehra, A., Ayala, P., Oliveira, L., He, J., Zakhidov, A.A., Howard, A., Wilkins, J., Rao, A.M. Magnetic properties of sulfur-doped graphene., J. Magn. Magn. Mater. 401, 70-76 (2016)

38. Nair, R.R., Sepioni, M., Tsai, I.-L., Lehtinen, O., Keinonen, J., Krasheninnikov, A.V., Thomsoin, T., Geim, A.K., Grigorieva, I.V.: Spin-half paramagnetism in graphene induced by point defects. Nat. Phys. 8, 199-202 (2012)

39. Ji, Z., Shen, X., Zhu, G., Zhou., Yuan, A.: Reduced graphene oxide/nickel nanocomposites: facile synthesis, magnetic and catalytic properties. J. Mater. Chem. 22, 3471-3477 (2012).

40. Dubey, R., Bajpai, J., Bajpai, A.K.: Green synthesis of graphene sand composite (GSC) as novel adsorbent for efficient removal of $\mathrm{Cr}(\mathrm{VI})$ ions from aqueous solution. J. Water. Process. Eng. 5, 83-94 (2015)

Publisher's Note Springer Nature remains neutral with regard to jurisdictional claims in published maps and institutional affiliations. 